\title{
IDENTIFICACION DE Fusarium solani (Mart.) Sacc. COMO AGENTE CAUSAL DE LA PODREDUMBRE DEL PIE DE TOMATE
}

\author{
(Identification of Fusarium solani (Mart.) Sacc. as the causing agent \\ of the foot rot in tomato)
}

\author{
J. Montealegre', S. Donoso ${ }^{1}$, R. Herrera ${ }^{1}$ \& X. Besoain ${ }^{2}$ \\ ${ }^{1}$ Universidad de Chile, Facultad de Ciencias Agronómicas. Santiago. \\ e-mail: jmonteal@abello.dic.uchile.cl \\ ${ }^{2}$ Universidad Católica de Valparaíso, Facultad de Agronomía. Quillota.
}

Palabras clave: Fusarium solani, podredumbre del pie y raíz del tomate, marchitez del tomate.

Key words: Fusarium solani, tomato, foot and root rot, tomato wilt.

\section{RESUMEN}

Se investigó la etiología de una pudrición basal y radical causada por Fusarium sp. en plantas de tomate enfermas en invernaderos fríos de la V Región de Chile. Se identificó como patógeno a Fusarium solani, hongo que ha sido descrito en otros países causando síntomas similares a los observados en este trabajo. Los sintomas en terreno y en las plantas inoculadas fueron: pudrición radical, podredumbre del pie y necrosis vascular.

\section{INTRODUCCION}

Debido a la condición de monocultivo del tomate, producido bajo invernadero en la V Región de Chile, sujeto a condiciones de alta temperatura y humedad, el cultivo está propenso a enfermedades fungosas que afectan el sistema radical, entre las que se encuentran las fusariosis.

Además de Fusarium oxysporum f. sp. lycopersici y $\boldsymbol{F}$. oxysporum f. sp. radicis-lycopersici, detectados a nivel mundial causando enfermedades en tomate, también se ha descrito a Fusarium solani como un importante patógeno de este cultivo en distintos lugares del mundo, entre los cuales se cuentan Argentina (Wolcan \& Lori, 1991), California, Australia, India, Turkía e Israel (Cucuzza \& Waterson, 1992 y Miyao et al., 2000).

En Australia, Fusarium solani es la principal causa de marchitamiento en tomates resistentes a Fusarium oxysporum f. sp. lycopersici (Vawdrey \& Peterson, 1988).

Durante la temporada 1999-2000, se detectaron en invernaderos fríos, plantas de tomate en la zona productora
ABSTRACT

The etiology of a foot and root rot caused by Fusarium sp. on tomato plants attacked by this disease in cold greehouses of the V region of Chile was investigated. Fusarium solani, a fungus that has been described in other countries as a causing agent of similar symptoms to those observed in this research was identified as a pathogen. Symptoms in field and in inoculated plants were: root rot, foot rod and vascular necrosis.

de la V Región de Chile, afectadas por una fusariosis que causaba principalmente "podredumbre del pie". Por tal razón, el objetivo de esta investigación fue determinar la etiología de esta enfermedad.

\section{MATERIALES Y METODOS}

Las cepas de Fusarium usadas en la investigación, correspondieron a aislamientos del hongo efectuados en agar papa dextrosa (APD), realizado a partir de plantas enfermas con síntomas de podredumbre del pie y además marchitez vascular, provenientes de invernaderos fríos de la V Región.

Para las pruebas de patogenicidad, se trabajó en macetas, con suelo fumigado con bromuro de metilo y plántulas de tomate (Lycopersicum esculentum L.) de variedades susceptibles y resistentes a Fusarium oxysporum f. sp. lycopersici (Tabla 1), las que se hicieron crecer bajo condiciones de invernadero a una temperatura media de 
Tabla 1. Respuesta a la inoculación con Fusarium solani de diferentes variedades de tomate resistentes a las diferentes razas de Fusarium oxysporum f. sp. lycopersici.

\begin{tabular}{|c|c|c|c|c|c|}
\hline \multirow{3}{*}{$\begin{array}{l}\text { Variedades de } \\
\text { tomate }\end{array}$} & \multicolumn{3}{|c|}{$\begin{array}{l}\text { Resistencia a Fusarium } \\
\text { oxysporum f. sp. lycopersici }\end{array}$} & \multirow{2}{*}{\multicolumn{2}{|c|}{$\begin{array}{l}\text { Respuesta de las diferentes variedades } \\
\text { a la inoculación con cepas de } F \text {. solani } \\
\text { Sistemas de inoculación }\end{array}$}} \\
\hline & \multirow[b]{2}{*}{ raza 1} & \multirow[b]{2}{*}{ raza 2} & \multirow[b]{2}{*}{ raza 3} & & \\
\hline & & & & $\begin{array}{c}\text { Inmersión de } \\
\text { raices }\end{array}$ & $\begin{array}{c}\text { Semillas de avena } \\
\text { colonizadas }\end{array}$ \\
\hline Bonny Best & $\mathbf{S}$ & $\mathbf{S}$ & $\mathbf{S}$ & $\mathbf{S}$ & $\mathbf{S}$ \\
\hline Manapal & $\mathbf{R}$ & $\mathbf{S}$ & $\mathbf{S}$ & $\mathbf{S}$ & $\mathbf{S}$ \\
\hline Horizon & $\mathbf{R}$ & $\mathbf{R}$ & $\mathbf{S}$ & $\mathbf{S}$ & $\mathbf{s}$ \\
\hline 13R-1 & $\mathbf{R}$ & $\mathbf{R}$ & $\mathbf{R}$ & $\mathbf{S}$ & $\mathbf{S}$ \\
\hline E 335 & $\mathbf{S}$ & $\mathbf{R}$ & $\mathbf{S}$ & $\mathbf{s}$ & $\mathbf{S}$ \\
\hline E 271 & $\mathbf{S}$ & $\mathbf{R}$ & $\mathbf{R}$ & $\mathbf{S}$ & $\mathbf{S}$ \\
\hline
\end{tabular}

media de $24^{\circ} \mathrm{C}$ y se regaron regularmente con agua potable, acorde con las necesidades de las plantas.

Para la inoculación de las plantas:

a.- Se efectuaron cortes de los ápices radicales de plántulas con 2 a 3 hojas verdaderas y luego se efectuó una inmersión de raíces durante 1 hora en una suspensión en agua destilada estéril con $10^{7}$ conidios $/ \mathrm{mL}$ obtenidas de un cultivo puro de una semana de edad en APD.

b.- Incorporación en el hoyo de trasplante de 10 a 15 semillas de avena (Avena sativa $\mathrm{L}$.) colonizadas con la cepa de Fusarium sp. respectiva. Para este efecto el hongo se hizo crecer en matraces que contenían semillas de avena previamente esterilizadas en autoclave a $120^{\circ} \mathrm{C}$ por 20 minutos por dos veces consecutivas. Estas fueron inoculadas con la cepa de Fusarium elegida y se dejaron crecer por 15 días a $22^{\circ} \mathrm{C}$.

Se inocularon veinte plántulas, dejándose también 20 controles. La evaluación consistió en cuantificar las plántulas muertas a los 30 días postinoculación, y en las que presentaban síntomas se reaisló el hongo en APD.

Se tomaron microfotografías y se hicieron mediciones de 100 conidios y clamidosporas, observándose además sus características morfológicas. Estas se obtuvieron de cultivos del hongo en APD de 10 días de edad. Paralelamente, se envió la cepa de Fusarium utilizada para las pruebas de patogenicidad al Commonwealth Agricultural Bureau International (CABIInglaterra) para su identificación.

\section{RESULTADOS Y DISCUSION}

Patogenicidad. Todas las variedades de tomate inoculadas que eran resistentes a $\boldsymbol{F}$. oxysporum f. sp. lycopersici fueron sensibles a la cepa estudiada con ambas técnicas de inoculación (Tabla 1), concluyéndose que el patógeno no correspondía a Fusarium oxysporum f.sp. lycopersici sino a Fusarium solani, de acuerdo a las observaciones morfológicas y a la identificación realizada en el CABI.

El hongo se reaisló a partir del pie y las raíces de todas las plántulas muertas o que presentaban síntomas de la enfermedad, resultando sus características morfológicas similares al inoculado.

Síntomas. Los síntomas observados en terreno y en las plantas inoculadas fueron, podredumbre del pie, pudrición radical y necrosis vascular, sintomas que coinciden con los descritos en la literatura (Vawdrey \& Peterson, 1988; Wolcan \& Lori, 1991 y Miyao et al., 2000), para los causados por Fusarium solani en tomates.

Además, se observó clorosis intervenal y blanqueamiento de la lámina foliar, la raíz primaria presentó lesiones corticales pardo rojizas y decoloración vascular sobre y bajo las lesiones; esto concuerda con lo descrito por Cucuzza \& Waterson (1992). Al mismo tiempo en la raíz se observó una necrosis y las raicillas se pudrieron.

En cortes longitudinales de los tallos, se evidenció en la médula una podredumbre seca de aspecto corchoso, lo que coincide con lo descrito por Wolcan \& Lori (1991).

El hongo en medio de cultivo APD presentó pigmentación pálida, celeste a azul, lo que coincide con Booth (1971). Las colonias eran de aspecto uniforme con micelio no denso, más elevado en el centro, lo que concuerda con lo observado por Wolcan \& Lori (1991). 
Tabla 2. Tamaño de esporas de la cepa de $F$. solani estudiada y comparada con lo reportado por Booth (1971).

\begin{tabular}{|l|c|c|c|c|c|}
\hline \multirow{2}{*}{ Referencias } & \multicolumn{2}{|c|}{ Microconidios } & \multicolumn{2}{c|}{ Macroconidios } & Clamidosporas \\
\cline { 2 - 6 } & ancho & largo & ancho & largo & diámetro \\
\hline Booth (1971) & $3-4 \mu \mathrm{m}$ & $6-9 \mu \mathrm{m}$ & $4,5-6 \mu \mathrm{m}$ & $35-55 \mu \mathrm{m}$ & - \\
\hline Cepa estudiada & $2-4 \mu \mathrm{m}$ & $2,7-8,9 \mu \mathrm{m}$ & $2,2-4,4 \mu \mathrm{m}$ & $9,1-38,9 \mu \mathrm{m}$ & $6,2-11,1 \mu \mathrm{m}$ \\
\hline
\end{tabular}

Características morfológicas de $F$. solani (Mart.) Sacc.

Morfológicamente se pudo observar que $\boldsymbol{F}$. solani presentó conidióforos largos (Figura 1) con abundantes microconidios de tamaño promedio de $2,4 \mathrm{x}$ $5,3 \mu \mathrm{m}$. Los macroconidios presentaron por lo general, 1 a 4 septos, su tamaño varió entre 2,9 y $4,2 \mu \mathrm{m}$ de ancho y 12,8 a $34,4 \mu \mathrm{m}$ de largo. En general los rangos de las medidas obtenidas tanto de los micro como macroconidios, fueron inferiores a las que señala Booth (1971). Las clamidosporas presentaron un diámetro promedio de $8,4 \mu \mathrm{m}$ (Tabla 2).

\section{CONCLUSIONES}

En base a los antecedentes expuestos, se concluye que la "podredumbre del pie del tomate" es causada por el hongo Fusarium solani.

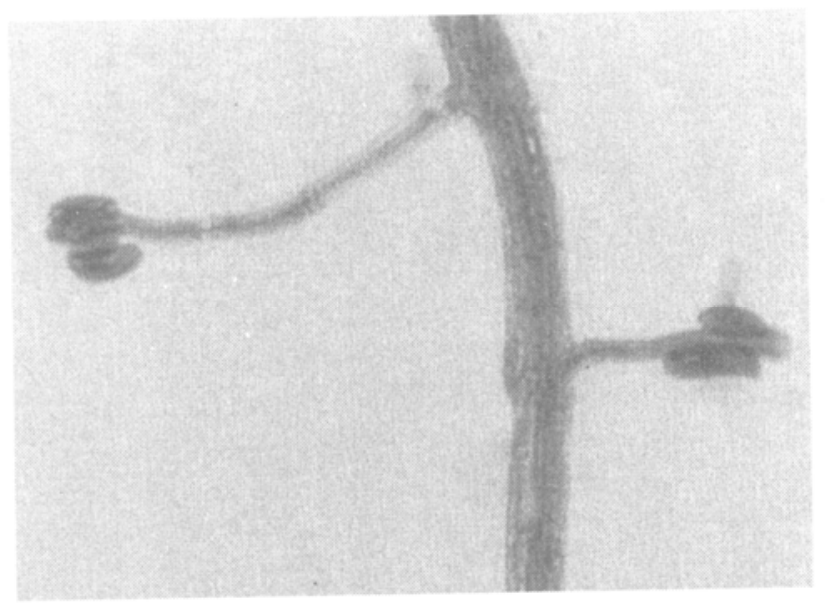

Figura 1.- Conidióforos, micro y macroconidios de $F$. solani $(640 \mathrm{X})$.

\section{REFERENCIAS}

Booth, G. (1971). Genus Fusarium. Commonwealth Mycologycal Institute, Kew, Surrey, England.

Cucuzza, J. \& Waterson, J. (1992). Foot rot of tomato caused by Fusarium solani in California. Plant Dis. 76: 101

Miyao, B., Falk, B.; Subbarao, K. \& Stapleton, J. (2000). Tomato Fusarium Foot Rot. U.C. Pest Management Guidelines. Disponible en: http://www.ipm.ucdavis.edu

Vawdrey, L. \& Peterson, R. (1988). Fusarium solani, the cause of root rot of tomatoes in Central Queensland. Australasian Plant Pathol. 17: 2425

Wolcan, S. \& Lori, G. (1991). Podredumbre del pie del tomate, causada por Fusarium solani (Mart.) Sacc. Revista de la Facultad de Agronomía, Universidad Nacional de La Plata 12:47-51 